This paper is a postprint of a paper published in IET Generation, Transmission \& Distribution and is subject to Institution of Engineering and Technology Copyright. The copy of record is available at IET Digital Library

'Moisture Ingress into Low Voltage Oil-Impregnated-Paper Insulated Distribution Cables'

IET Proc Sci., Meas. \& Tech. 1 Issue 5 (2007) 276-283

$M$ Wang, S M Rowland and P E Clements

Digital Object Identifier: 10.1049/iet-smt:20060134

\title{
Moisture Ingress into Low Voltage Oil-Impregnated-Paper Insulated Distribution Cables
}

\author{
M. Wang, S. M. Rowland and P. E. Clements \\ The School of Electrical and Electronic Engineering \\ The University of Manchester \\ PO Box 88, Manchester, M60 1QD, UK
}

\begin{abstract}
Investigations into the effect of moisture on the degradation of oil-impregnated-paper insulated low voltage cables has shown that moisture ingress plays a key role in cable failure, and that the ionic content of the moisture is critical. Here moisture ingress into oil-impregnated-paper insulated cable is studied in the laboratory. Experiments have been performed in which cable samples have been exposed to moisture for varying periods, allowing the progress of moisture diffusion within the insulation to be studied. Karl-Fischer titration and observation of dye movement have been used to measure moisture transport. Moisture within a 'wet cable' is shown to be non-uniformly distributed within a sector-shaped core, and dependent on the temperature of the cable. Measurements of insulation conductivity are presented illustrating the resulting macroscopic changes to insulation properties for comparison. An increased rate of penetration of moisture into the cable core results at elevated temperatures, whilst saturation levels are reduced.
\end{abstract}

Index Terms - Oil/paper insulation, underground distribution cable, moisture, ageing, LV fault. 


\section{INTRODUCTION}

Cost-effective management of low voltage (LV) distribution cables presents significant challenges because they have low value per unit length, carry relatively low levels of power to few customers, and are disparate assets, spread throughout a network. Cables in the UK typically have a range of ages up to, and over, forty years, and have a number of designs [1]. Understanding the nature of LV failure is key to improving network reliability. The many detailed studies of medium and high voltage (HV) cables are not readily transferable, since failure occurs in LV cable insulation at fields over an order of magnitude lower than in HV cables [2]. Generic characterization of an oil-impregnated-paper (OIP) insulated cable is not easy, since a network may contain cables installed at any time over the past hundred years, and materials used and quality control may have varied radically over this period. Studying microscopic material properties may, therefore, not be as fruitful as considering macroscopic generic properties such as moisture transport and conductivity [3].

Work presented here is building towards the development of tools to more efficiently manage LV distribution cables. Moisture diffusion is studied by dissecting LV paper-insulated, leadcovered (PILC) cable insulation. Investigation into the movement of moisture in OIP insulated LV cable cores shows that moisture ingress is far from uniform. The combination of KarlFisher (KF) titration and tracer dyes is used to fully identify the distribution of moisture in the cable.

It is generally accepted that the presence of moisture in oil-based dielectrics is harmful $[4,5]$, and moisture ingress affects the dielectric properties of OIP cable insulation [6,7]. The change in an insulation material due to moisture can be quantified by dielectric response in the time or frequency domain. For example, dielectric loss increases and dielectric breakdown strength decreases as moisture content rises, leading to failures in OIP cable systems [8]. In addition, 
other processes such as corrosion of metal elements are accelerated by the presence of moisture. The progress of water ingress is not well studied, and very little work has been reported on low voltage distribution cable failure. The intention of the experimental work here is to understand the diffusion of high levels of water content (up to $25 \%$ by weight) and so gain an insight into its influence on the dielectric strength of OIP insulation in low voltage applications.

\section{EXPERIMENTAL}

\subsection{LOW VOLTAGE CABLE GEOMETRY}

The LV PILC cable sample tested in the laboratory is unused $185 \mathrm{~mm}^{2} \square$, 4-core, stranded aluminium, OIP insulated cable. Figure 1 illustrates the cable structure. In practice, moisture is likely to originate after damage to the lead sheath. Such damage may arise from corrosion or previous accidental dig-up. For the purpose of studying moisture ingress through the insulation, the work reported here is focused on the sectorial shaped stranded aluminium conductor and associated helically wrapped OIP layers only.

The insulation around each sectorial-shaped core is formed by seven wound OIP insulation layers, forming insulation $0.89 \mathrm{~mm}$ thick. The design electrical stress across the insulation in service is $415 \mathrm{~V}$ over $1.8 \mathrm{~mm}$, giving $230 \mathrm{~V} / \mathrm{mm}$, much lower than the 2 or $3 \mathrm{kV} / \mathrm{mm}$ seen in HV cables. OIP layers overlap by $25 \%$ to $50 \%$, and a small slot (about $1 \mathrm{~mm}$ wide) results between adjacent paper strip edges on the same layer. This is shown in Figures 2 and 3. Figure 3 shows the paper layers of insulation unwrapped from the cable. D1 shows the axial direction, and D2 the circumferential direction. The outer layer of paper is at the top of the picture and the inner layers have yet to be fully unwrapped. Regions A1, B1 and C1 etc. are those associated with the relatively flat parts of the cable cross-section shown in Figure 2. Figure 2 also identifies the helical slot between the adjacent layers' edges, labelled L1 and L1', of the outermost layer of paper. 


\subsection{GEOMETRY ASSOCIATED WITH THE UNWOUND INSULATION}

Figure 4 illustrates the geometry of the unwound OIP insulation from a single core. The analysis areas and boundaries are defined for later discussion. In this schematic diagram of the unwrapped insulation, the area enclosed by points EFGH represents one section of a paper tape forming a layer other than the most inner or outer. The equivalent areas of tapes outside and inside (or above and below) the tape under consideration are enclosed by areas the IJKL and MNOP respectively. The bold lines, for example UV, represent the centres of the three regions of highest curvature on the conductor cross-section. The arrow D2 represents the circumferential direction, which is perpendicular to UV and the axial direction D1 of Figure 3. The sectorial shaped conductor contains three relatively flat areas marked as A1, B1, C1, A2, B2... (also shown in Figures 2 and 3). The section of edges of the paper tape marked L1 and L1' lie together in the cable to form a region of slot S1. In other words, L1 and L1' directly face each other with the slot S1 in between when they are in the original helically wrapped condition. Similarly, L2 and L2' share the same slot region S2, and L3 and L3' share the same slot region S3. When it is in the original wrapped condition, the tape EFGH of the layer under consideration is directly covered by the next tape up IJKL; the area efgh is covered by the area $i j k l$ of the upper layer, forming the slot above the line ef. Likewise, the whole of the wrapping arrangement can be derived for the full seven helically butted insulation layers.

\subsection{CABLE SAMPLE PREPARATION FOR MOISTURE INGRESS TESTS}

For simplicity cable cores are studied in isolation, and these are carefully removed from complete cable structures. Two modes of soaking the samples were employed at room temperature. Firstly a uniform ingress test was used in which a large surface area was exposed to water. Alternatively a localized ingress test was used where moisture passed through a defined hole in a moisture-proof sheath. Before soaking, the two ends of each sample were 
sealed with epoxy resin in order to minimize effects other than moisture ingress from the outside layer towards the core. This is shown in Figure 5. For the localized ingress test, the samples were wrapped tightly in aluminium foil with a $15 \mathrm{~mm}^{2}$ hole in the foil on the cable surface. Samples were then soaked individually in tap water (conductivity $90 \pm 10 \mu \mathrm{S} / \mathrm{cm}$ ). In some of these tests a commercial blue dye was added to the tap water. The conductivity of the mixture of dye and tap water was $300 \pm 50 \mu \mathrm{S} / \mathrm{cm}$.

\subsection{TESTING AT ELEVATED TEMPERATURE}

To allow elevated temperature measurements, the temperature of the core was controlled by insertion of thermostatically controlled soldering irons directly into the centre of each end of each core. In these cases the central section of the core length was exposed to moisture by inserting the length into a waterproof box with appropriately sized holes in opposite sides. The cable was sealed into the box with epoxy resin. The box was then filled with water as required. $140 \mathrm{~mm}$ of cable was exposed to moisture in this manner.

Figure 6 is a schematic diagram of the test arrangement. This test was used to simulate moisture diffusion occurring under conditions where a large part of the insulation surface is in touch with moisture due to damage of the cable sheath. Test details have been described previously [9]. Measurements were taken with immersion in water at room temperature and with the core directly heated to $75^{\circ} \mathrm{C}$. In the latter case, the water at the cable surface reached equilibrium at $65^{\circ} \mathrm{C}$ after two hours.

To further clarify the effect of temperature, samples of paper from the innermost and outermost layers were soaked directly in water at $20^{\circ} \mathrm{C}, 65^{\circ} \mathrm{C}$ and $75^{\circ} \mathrm{C}$ for comparison. This was done by directly immersing samples of unwrapped OIP in water to determine their moisture uptake. The results of these tests are given in Table 1. Table 2 shows the same experiments but with the layer under test left on the core, e.g. removing the outer layers to 
directly expose inner layers to moisture. In both these cases the elevated temperature is reached by heating the water rather than heating the core.

\subsection{MEASUREMENTS OF LEAKAGE CURRENT}

An investigation was also carried out into the insulation resistance and the corresponding leakage current passing through the dielectric during a set of uniform soak tests [10]. After the cable samples have been soaked in tap water for assigned periods, they were taken out for immediate measurement of leakage current flowing through the OIP insulation. Figure 7 shows the schematic diagram of the test circuit. In trials no difference was observed when guard rings were used in the test circuit. Since any interference with the surface was likely to promote damage and further moisture transport, the measurements were done without the presence of guard rings.

Voltages between $40 \mathrm{~V}$ and $240 \mathrm{~V}$ AC were applied to the dielectric between the conductor and an earthing electrode. The surface area of the earthing copper electrode, which was wrapped onto the core was $1034 \mathrm{~mm}^{2}$. Two soaked cable samples were used each day for each measurement. The leakage currents were measured at different locations along the cable samples, and an average value was obtained for each specific voltage at a defined soaking time.

\subsection{KARL-FISCHER TITRATION MEASUREMENTS}

After the samples were soaked, they were taken out of the water for immediate moisture content (MC\%) measurement by the Karl Fischer (KF) technique. The size of each paper sample for the KF measurement was approximately $20 \mathrm{~mm} \times 24 \mathrm{~mm}$. This insulation area is encompassed by the sections A1, A2 and A3 of Figures 2 and 4 for each paper layer. Before cores were dissected, pin-holes were created which penetrated through the insulation close to the edges of area 'A' (referred to in Figures 2 and 4) as reference points, in order that samples could be taken from the same position of the conductor's insulation on consecutive layers for 
evaluation, shown in Figure 8. For each KF measurement, an OIP layer was peeled off and cut up before sealing the sample in a vial to maintain the original moisture and to minimize the loss through evaporation. Three samples were taken from each layer in order to get an accurate value of the moisture content, and also to investigate whether or not the moisture absorption was uniform within the different layers.

\section{RESULTS}

\subsection{DETERMINATION OF UNIFORM MOISTURE INGRESS USING DYE}

After a core had been soaked, OIP layers were peeled off and arranged on a flat surface maintaining their original relative positions. Examples of the uniform ingress test with the dye are shown in Figure 9 to illustrate the manner of general moisture diffusion. The paper at the top of Figure 9 is that from the outer layer of insulation, and the paper at the bottom is from the innermost insulation. In general, with increased soaking time in the water, the dye extends further into the insulation, and covers a greater percentage of the circumference. The density of colour content also increases with time. Distinct regions of colour distribution can be observed, mainly on the three flatter regions around the sectorial shaped conductor (A1, B1, C1 etc). The dye is generally absent from the three very curved sections particularly further down the insulation layers. This indicates moisture cannot move circumferentially around the cable between these regions. The regions of dye, which extend parallel to the conductor, are mainly attributed to axial and radial diffusion towards the inner layers without circumferential diffusion. There is no evidence of moisture leaking in from the epoxy covered ends. Both pictures in Figure 9 show moisture ingress furthest into the cable in the centre of the cable length. Within each layer and each region $\mathrm{A}, \mathrm{B}$ and $\mathrm{C}$, moisture appears to be uniform. The long dimension of each dyed area seen in Figure 9 has a fixed angle to the paper edge, related to the helical layer wrapping geometry, and previously identified as D1. 
Each layer reveals narrow, straight lines of high dye density on the OIP layers when they are unwrapped from the conductor. These lines are attributed to the water and dye entering the small slot, between paper edges formed helically around the conductor. This is on the line equivalent to JK on the top surface and MP on the bottom surface of the tape length EFGH of Figure 4. These lines tend only to be within the regions A, B or C and do not normally extend into the section of insulation on the curved surface of the conductors.

In order to investigate axial and circumferential moisture penetration, holes were drilled vertically through the insulation of a cable core. This allowed moisture direct access to every layer of the insulation. Figure 10 reveals the moisture penetration after soaking such a sample for 3 days. The distribution of dye around each hole location extends in all directions and is roughly circular, revealing the diffusion rates in the circumferential direction are similar to the axial direction. In some cases, the hole crossed a slot between the paper tapes, which led to rapid diffusion down the slot, e.g. extending into region $\mathrm{A}, \mathrm{B}$, or $\mathrm{C}$, although not crossing their boundaries.

\subsection{LOCALIZED MOISTURE INGRESS}

By reference to the insulation geometric layout established in Figure 4, it is possible to analyze moisture ingress further. For example, if there is a localized moisture reservoir located at the centre of the area A2, on top of the layer in question, the moisture will diffuse in all directions but potentially at different diffusion rates. When the moisture reaches the paper tape edge (L4), where it meets its own edge (L4') again in the same layer, it will go into the helical slot between the two edges and may spread out along the slot rapidly if the slot is not fully filled with oil. This slot is bounded above and below by paper tapes, so the moisture can diffuse through the slot to the lower surface of the paper, and/or along the slot to enter the other areas that are connected to the slot. Thus, if the slot is not fully filled, the moisture diffuses through 
the slot S4 to the underneath layer part of A2, and/or transversely across the slot S4 to the area A3 of the same layer. Similarly, when the moisture reaches the boundary line L1', it would most likely diffuse through the slot $\mathrm{S} 1$ to the underneath of $\mathrm{A} 1$, and/or transversely cross the slot $\mathrm{S} 1$ to the area $\mathrm{A} 1$ of the same layer level.

It appears that the moisture does not significantly traverse through the OIP, but moves faster over the surfaces of the paper strips and in the slots formed by the helical paper edges within each layer. Between layers of paper two moisture diffusion directions are defined: the direction D1, the longitudinal direction of the conductor; and the direction D2, which is circumferential. Illustrations of how the moisture diffuses are shown in Figure 11. These pictures come from the localized ingress test samples, which are wrapped in aluminium foil in which a hole is made before soaking. Case A in Figure 11 shows two regions of moisture: the smaller one in the second layer was directly below the larger one in the outer layer before the cable was unwrapped. Case B shows the broader areas of moisture which have spread along the cable within a layer as well as moving to the next layer down. It is suggested that the slot played a major role in the process of moving the moisture to the layer beneath. Moisture has not reached the third layer in either case. It can be concluded that the radial diffusion depends upon a combination of axial and circumferential diffusion.

\subsection{CHANGES TO INSULATION RESISTIVITY}

Changes to the insulation resistance and the corresponding leakage current passing through the dielectric during a set of uniform soak tests without dye, are summarized in Figure 12. The leakage currents increase almost linearly with increasing soak time in the voltage ranges.

The main evidence of physical changes to a cable core after soaking is that the paper layers become slightly loose. The reason for this is not clear, although it is possible that oil has been 
displaced allowing space to develop inside the cable, or the water has allowed the paper to swell and elongate.

\subsection{MOISTURE CONTENT MEASUREMENT}

The moisture content (MC) for the original untreated and unaged 'dry' OIP layers in uncontrolled laboratory conditions is between $0.1 \%$ and $0.5 \%$. Measurements for soaked samples with and without dye were carried out. Figure 13 summarizes the moisture content values for the different layers under the uniform ingress test condition. Each value is the average of six samples of KF measurements generated from tests with and without dye under the same testing conditions. In this figure the maximum and minimum values recorded in each situation are shown along with the average. Figure 14 provides a comparison of data between different insulation levels. For each layer a set of measured moisture levels is shown after one, two and three days soaking at room temperature. Measurements are given for both the cases where dye was and was not used. This enables a vision on the general progress of moisture diffusion to be generated.

At low moisture levels the measured moisture content of each layer from tests with dye is typically higher than from tests without. This is because the moist part can always be located and measured when dye is present, whilst the samples taken from dye-free solutions are not necessarily from the most moist part, due to the invisibility of water ingress. As a result, the MC\% measured from tests with dye show more consistent and reproducible results than from those without. The amount of moisture through the insulation can be clearly seen, and its progress is rapid. For example, after one day's soaking, a moisture level greater than $5 \%$ is already seen in the fifth layer. With the prolonged soaking times, the $\mathrm{MC} \%$ values become more consistent, showing less variation between samples. 
Figures 15 and 16 show data from room temperature, and elevated temperature, testing over longer periods. No tracer dye was used in these tests. Each of the data points on the graphs is an average of 6 measurements, each of which has an error of $\pm 5 \%$. Also shown are the maximum and minimum measurements in each case. The different time values represent different core specimens being dissected. Thus the graphs do not track moisture content at a single place through time.

\section{MODELLING MOISTURE DIFFUSION}

The modeling of non-equilibrium transport by diffusion may use Fick's second law [11], whose numerical solution [12] to the differential equation may be written as:

$$
\frac{C_{s}-C_{x}(t)}{C_{s}-C_{0}}=\operatorname{erfc}\left[\frac{x}{2 \sqrt{D t}}\right]
$$

where $C_{0}$ is the concentration within the cable at the start of the process $(t=0), C_{x}(t)$ is the concentration at position $x$ at time $t$, in this case at the cable surface, the maximum saturation value is $C_{s}, D$ is the diffusion coefficient and 'erfc' is the complementary or Gaussian error function.

No good fit can be found to the data of Figures 15 and 16 using equation 1, with fixed values of $D$ and $C_{s}$. The uniform, room temperature samples can provide a reasonable fit, but only if the value of the diffusion coefficient is allowed to vary significantly through the material as shown in Figure 15. Figure 16 suggests a clear variation of $C_{s}$ through the elevated temperature sample, and the curves fitted allow this variation, but have fixed D at a value of 3.5 , the choice of which is fairly arbitrary, but chosen as a mid-value of those used in Figure 15.

An empirical exponential description of the form

$$
C_{x}=C_{s}\left(1-e^{-t / \tau}\right)
$$

has previously been found to provide a fit equally as good, where $\tau$ is a quadratic of $x$ [13]. 


\section{DISCUSSION}

Table 1 suggests that OIP stripped from a cable does have a temperature-dependent saturation level. However the variation is only $2 \%$ lower at $75^{\circ} \mathrm{C}$ than at $20^{\circ} \mathrm{C}$, and with the significant errors and standard deviation in the measurements, this can not be considered proven. The change with temperature is less than suggested by the curves of Figures 15 and 16, in which the core at $75^{\circ} \mathrm{C}$ appears to saturate at around $10 \%$ rather than $\sim 20 \%$ at $20^{\circ} \mathrm{C}$. Table 2 also shows a much larger variation for samples soaked on the core, the outermost layer changes from $23 \%$ at $20^{\circ} \mathrm{C}$ to $18 \%$ at $75^{\circ} \mathrm{C}$, and the innermost from $21 \%$ to $15 \%$ respectively. This difference in uptake accounts for the drop in saturation levels seen in the outer layer at the elevated temperature when the core is heated. However, this test does not fully account for the change seen in the inner layer which, when soaked with the outer layers intact and with the core directly heated, rose to only $10 \%$.

We suggest therefore that the method of heating may be critical to moisture uptake, and that heating externally may cause higher uptake at the core centre than heating the core directly. It also appears likely that disassembling the core paper layers makes them prone to taking up more moisture. For this reason when the inner layers are directly exposed they absorb more moisture than otherwise.

It is also suggested that the inner layers are different from the outer layers, because of the manufacturing processes. For example the laying-up tension and impregnation processes on manufacture will not be uniform through the sample. The variation in the amount of paper overlap of between $25 \%$ and $50 \%$ demonstrates a lack of control in the manufacturing of the cable core insulation. In these processes it is quite likely that the inner layers have a greater grease density and are compressed by the outer layers, and so do not take up as much water. Nor is the migration of water between the paper layers as easy toward the centre. 
From the experimental data, the correlation between moisture content measured by the Karl Fischer technique and the apparent relative indication shown by dye density is good. The test methods provide consistent and reproducible results, and the relative strengths of each can be used to quantify and trace moisture ingress. Using the two techniques together is an effective way to trace regions of high moisture content. Two key assumptions have been made in using the dye: firstly, that the presence of the dye does not affect the rate of moisture diffusion, and secondly, that the dye accurately portrays the penetration of moisture [14,15]. Certainly the make-up of the moisture and contaminants should be of concern since real underground water contains a variety of contaminants, including surfactants, which may enhance moisture ingress.

The cables examined suggest that moisture diffusion can easily penetrate a cable core in the axial direction by moving between the surfaces of the OIP via the slot within the helically wrapped layers. It is much more difficult for moisture to directly diffuse radially to the underneath layer through the OIP layer itself, due to the nature of OIP, whose density and impermeability are very high [16].

That the moisture moves between the paper layers quicker than through them indicates that the rate of moisture uptake by the paper itself may be slower than the movement of moisture into the cable. This may be the reason why the simple expressions used to model moisture transport do not readily yield good results, since both moisture transport and moisture absorption need to be considered. Essentially we are observing a composite system rather than a homogeneous material. As discussed previously there are sensible physical reasons to accept that the material properties, expressed by $D$ and $C_{s}$ do vary through the cable core. This might be due to oil penetration during manufacture or paper wrapping tension.

It is clear that a distinction must be seen between moisture penetration into the cable between paper layers and moisture absorption by the paper, as measured by the Karl Fischer technique. 
Further work is required to understand the implications of this in terms of the dielectric behaviour of the cable. Figures 15 and 16 and Tables 1 and 2 show that an elevated temperature has a measurable effect on the transport of moisture in the cable. In particular although saturation levels are reduced the speed of transport is higher. In service, a cable's temperature will vary with load and a further study of dynamic behaviour would be of considerable interest.

Once cable insulation has been thoroughly wetted, its physical structure is never as compact as when originally fabricated. Therefore, it can be concluded that changes in original insulation structure may lead to severe degradation in insulation property, which makes it easy for repeated moisture penetration and later conductive path formation. Further experimentation is needed to understand the impact of temperature cycling, electric fields and insulation leakage currents on moisture diffusion through OIP. The work here has shown that despite the inhomogeneity of the insulation system, the progress of moisture through the insulation may be reproducible and predictable in controlled circumstances.

\section{CONCLUSIONS}

The use of dye in water is a useful way to trace moisture diffusion through OIP insulation, by visual inspection. Combined with Karl Fisher titration the progress of water can be traced and quantified through a cable using destructive tests. Moisture diffusion is not uniform within a sector-shaped core. In particular, lower concentrations of water are found in the paper in regions with low radii of curvature around the core. The likely reasons for this are associated with paper wrapping tension and oil content.

Moisture can readily diffuse in slots formed between paper edges, and that diffusion tends to occur between paper surfaces rather than through their bulk. The helical butt-gap slots formed 
within the paper layers are critical to the diffusion of moisture both along the cable and through the insulation system.

Elevated temperature is shown to increase the speed of moisture transport to the inner layers, but reduce the saturation levels within the core. Further work is required to link the moisture diffusion process to cable failure, but the evidence produced suggests that this macroscopic modelling of behaviour may yield a deterministic model of low voltage failure in the future.

\section{ACKNOWLEDGMENT}

The authors are grateful to EDF Energy for the funding which enabled this work to be carried out.

\section{REFERENCES}

[1] Moore, G.F, Ed., 'Electric Cables Handbook', (Blackwell Science, 3rd edn, 1997)

[2] Rowland, S.M., Wang, M., and Van Luijk, N.G., 'Mechanisms of failure in oil and paper insulated low voltage distribution cables', 3rd IEE International Conference on Reliability of Transmission and Distribution Networks, London, England, 2005, pp. 167-171

[3] Cherukupalli, S., Buchholz, V., Colwell, M., Crine, J.P., and Keefe, R.J., 'Condition assessment of distribution PILC cables from electrical, chemical, and dielectric measurements', IEEE Electrical Insulation Magazine, 2004, 20, (4), pp. 6-12

[4] Zaengl, W.S., 'Dielectric spectroscopy in time and frequency domain for HV power equipment, Part I: theoretical considerations', IEEE Electrical Insulation Magazine, 2003, 19, (5), pp. 5-19

[5] Gubanski, S.M., and Boss, P., 'Dielectric response methods for diagnostics of power transformers', IEEE Electrical Insulation Magazine, 2003, 19, (June), pp. 12-18 
[6] Neimanis, R., Saha, T.K., and Eriksson, R., 'Determination of moisture content in mass impregnated cable insulation using low frequency dielectric spectroscopy', IEEE Power Engineering Society Summer Meeting, Seattle, USA, 2000, pp. 463-468

[7] Neimanis, R., and Eriksson, R., 'Diagnosis of moisture in oil/paper distribution cables-Part I: Estimation of moisture content using frequency-domain spectroscopy', IEEE Trans Power Delivery, 2004, 19, (1), pp. 9-14

[8] Densley, J., 'Ageing mechanisms and diagnostics for power cables - an overview', IEEE Electrical Insulation Magazine, 2001, 17, (1), pp. 14-22

[9] Rowland, S. M., Wang, M., and Michel, M., 'Effect of moisture diffusion and heat cycling on low voltage oil-impregnated-paper insulation distribution cables', IEEE Conf Electrical Insulation and Dielectric Phenomena, Kansas City, 2006, pp. 237-240

[10] Wang, M., Rowland S. M., and Van Luijk N. G., 'The influence of moisture on low voltage oil-and-paper insulated distribution cables', IEEE Conf Electrical Insulation and Dielectric Phenomena, Nashville, 2005, pp. 112-115

[11]Shewmon, P., 'Diffusion in Solids', (The Minerals, Metals \& Materials Society, Diffusion in Solids. 2nd edn. 1989), pp. 10-23

[12] John, V., 'Introduction to Engineering Materials', (MacMillan, London, 3rd edn.1992), pp. $158-159$

[13] Wang, M., Rowland, S. M., and Clements, P., 'A mechanism of failure of oilimpregnated paper low-voltage cables', $10^{\text {th }}$ International Electrical Insulation Conference, Insucon, Birmimgham, 2006, pp. 178-182

[14] http://www.tramfloc.com/tf74.html, accessed February 2007

[15] http://www.brightdyes.com/index.html, accessed February 2007 
[16] Richard, M.E., 'Handbook of Physical and Mechanical Testing of Paper and Paperboard', Vol. 2, (Marcel Dekker, 1984), pp. 216-218

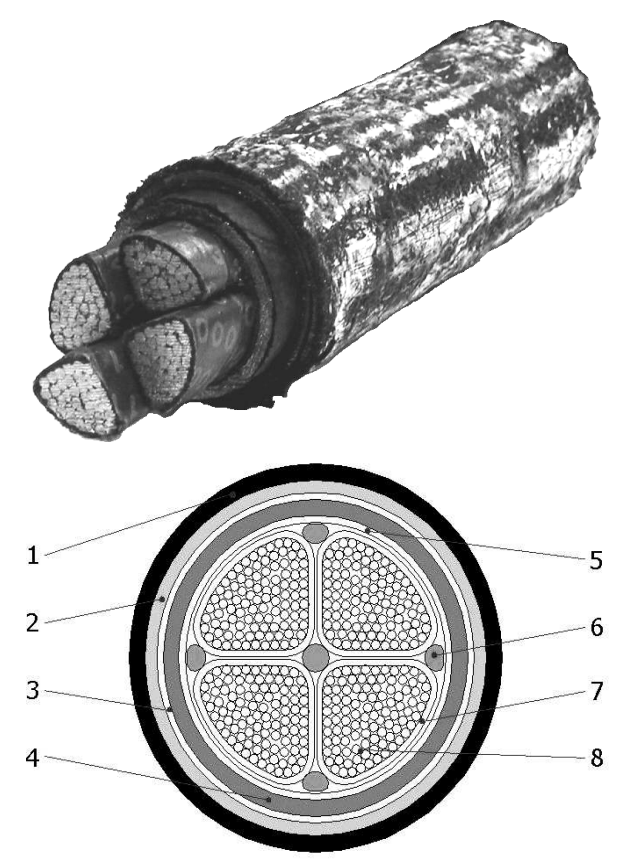

Figure 1. LV cable structure and cross-section: 1. bituminized outer cover, 2. steel armour, 3. OIP insulation layer, 4. lead sheath, 5. OIP belt insulation, 6. fillers, 7. OIP core insulation, 8. compacted stranded aluminium conductor. 


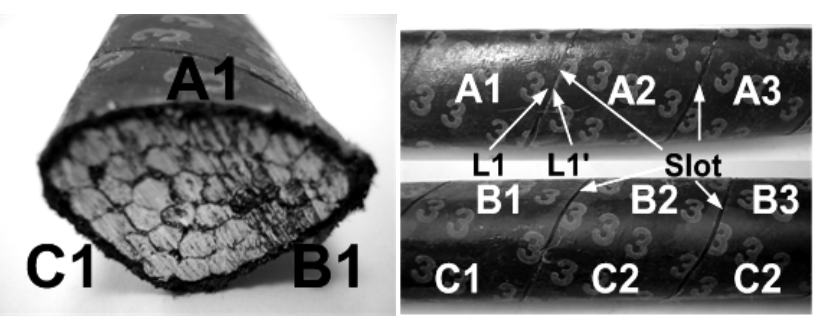

Figure 2. LV cable core and dielectric.

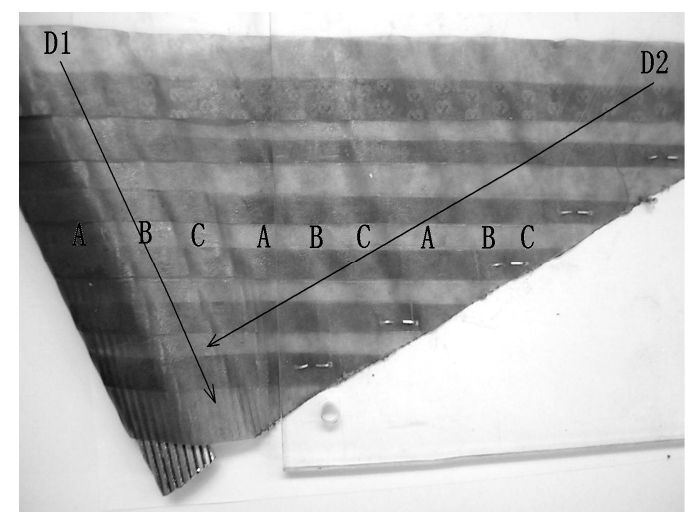

Figure 3. Dissection of the OIP insulation of one PILC cable core.

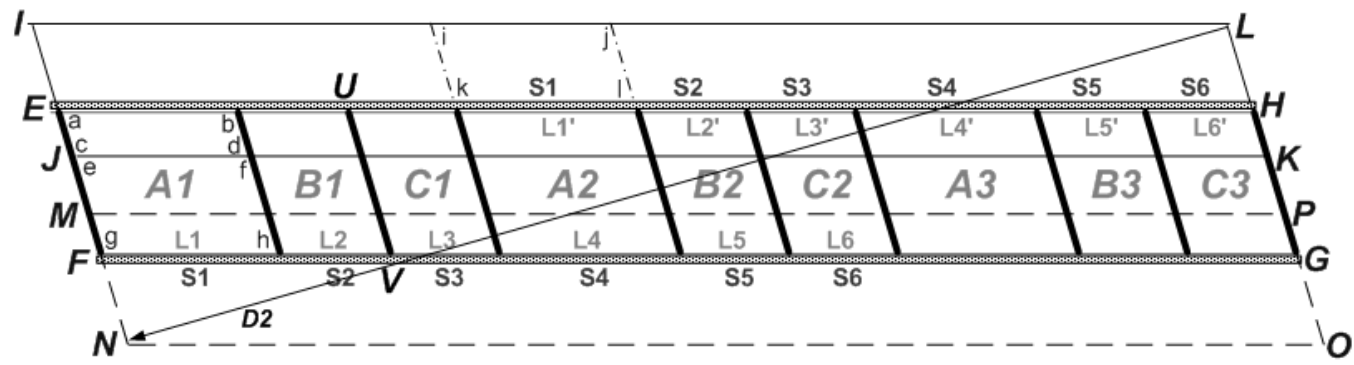

Figure 4. Description used to assist understanding of the unwrapped insulation. 

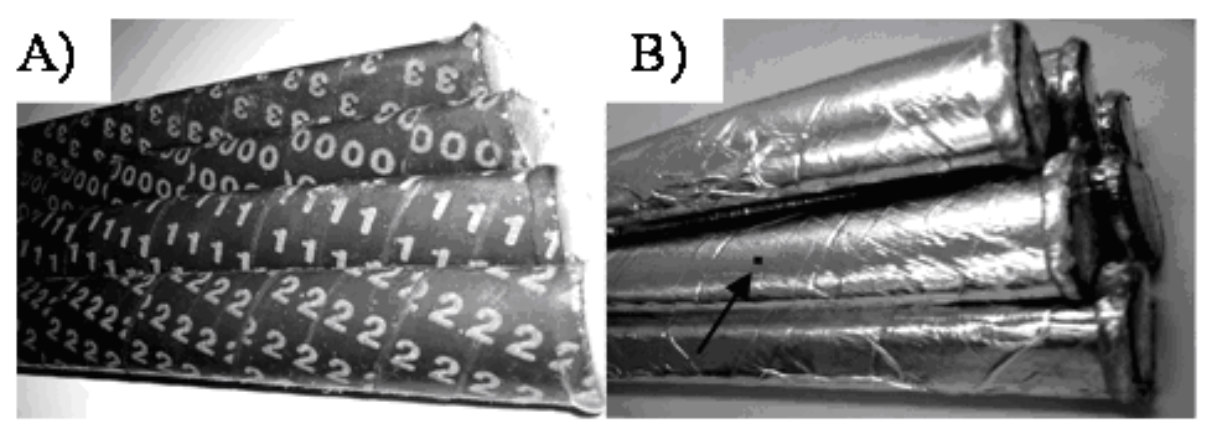

Figure 5. Sample preparation for uniform and localized ingress tests: A) for uniform diffusion test and B) for a local diffusion test. The arrow indicates the location of a hole in the moisture barrier.

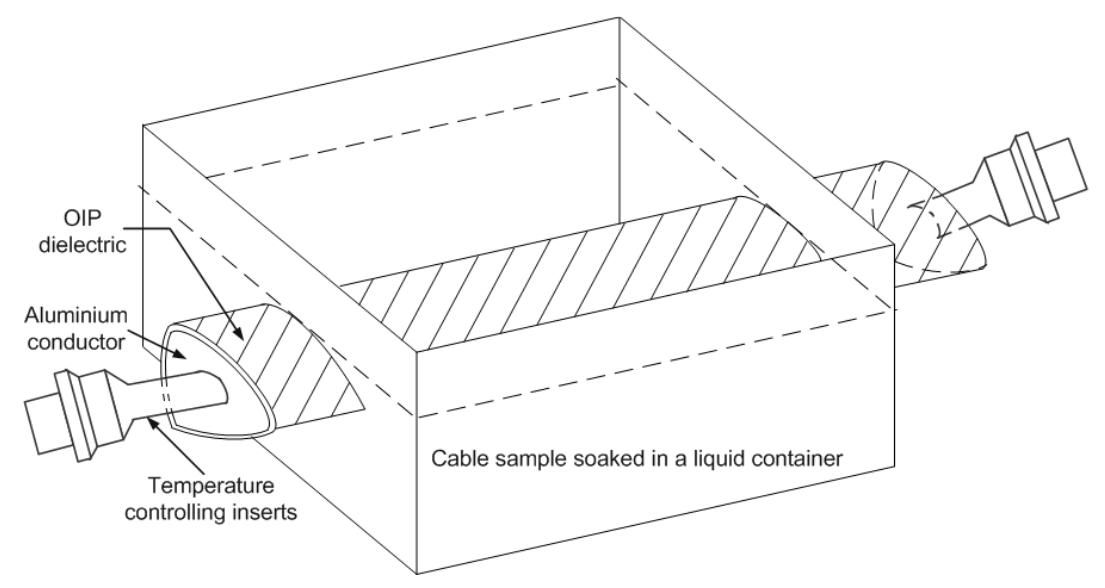

Figure 6. Cable core soaking in water with an elevated core temperature

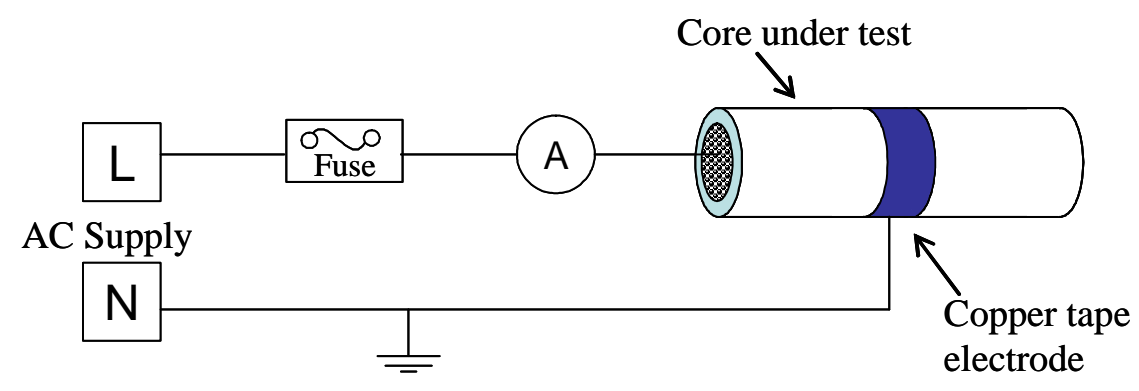

Figure 7. Schematic diagram of leakage current measurement through the cable dielectric 


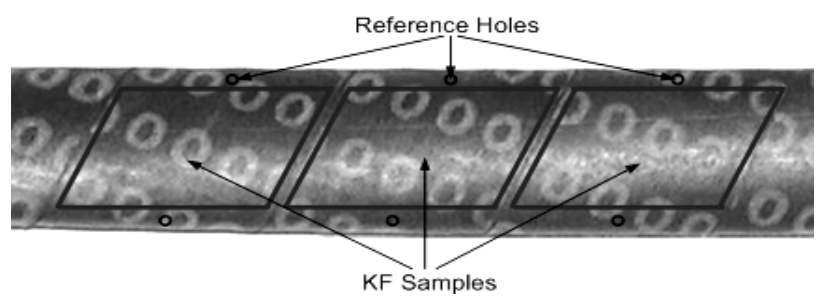

Figure 8. Sampling from the core insulation for moisture content measurement

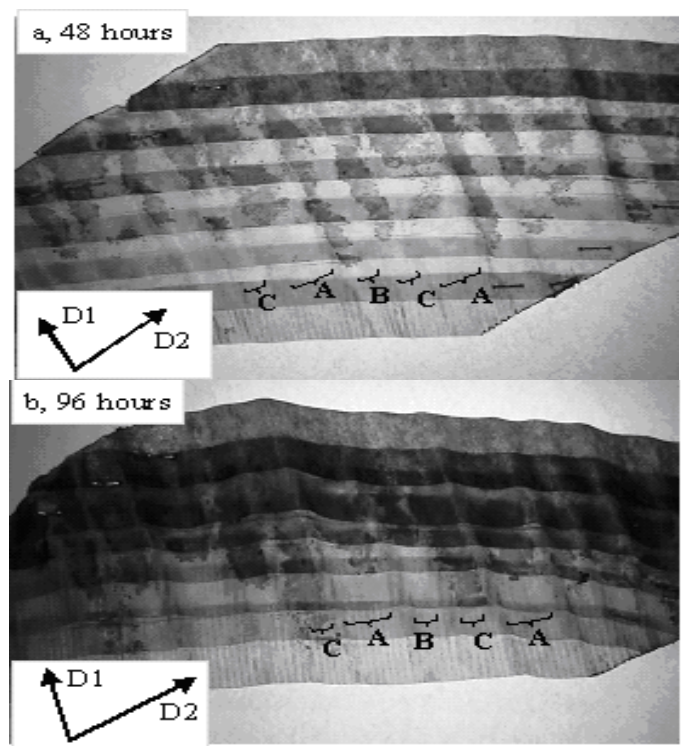

Figure 9. Mass moisture ingress evidence after 48 and 96 hours. Dark strips running left to right are the areas of paper overlap. Dark, irregular regions running top to bottom are dyed areas of moisture. 


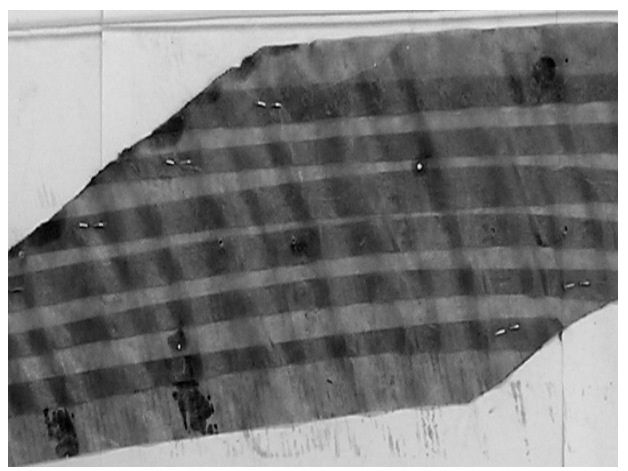

Figure 10. Moisture penetration through the insulation layers after soaking for three days.

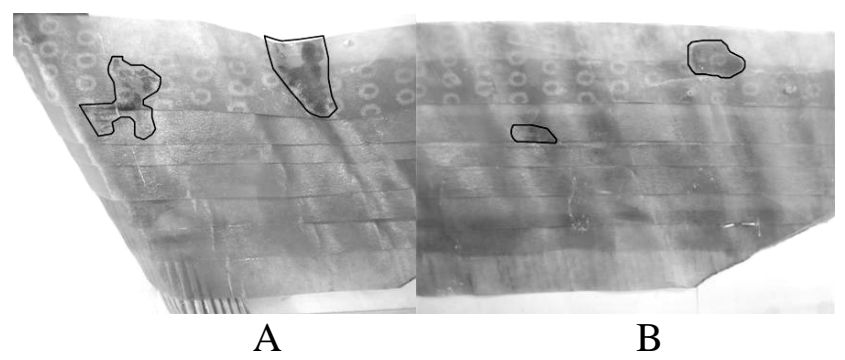

Figure 11. Dye ingress from a surface opening. Areas of dye have been outlined for clarity. After: A) 60 and B) 15 days.

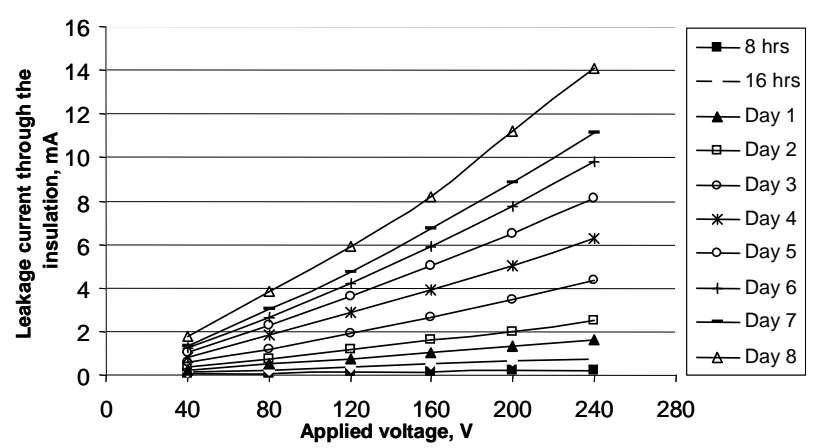

Figure 12. Insulation leakage current as a function of time in soak. 


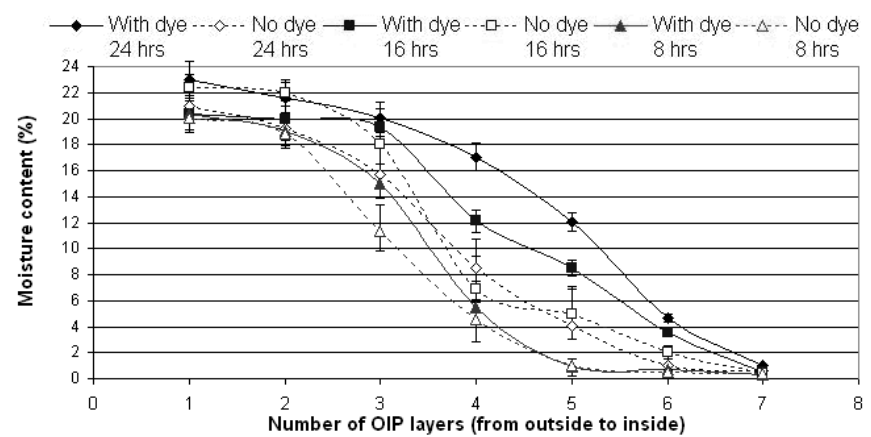

Figure 13. Moisture content of each OIP layer for different groups of tests. Errors of moisture content are $\pm 5 \%$ of the measured value. The maximum and minimum recorded values for each situation are shown with the average.

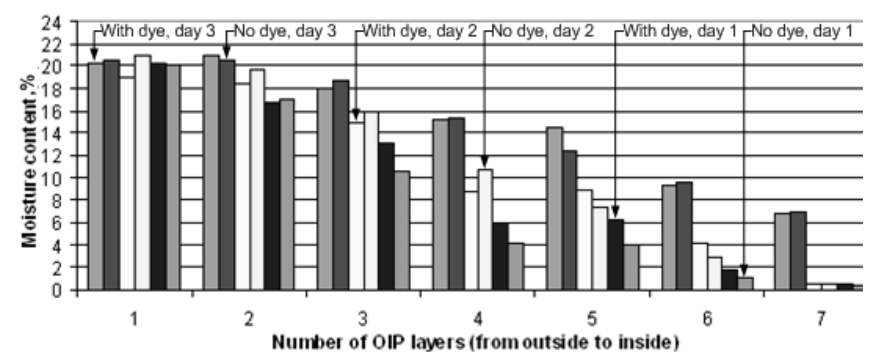

Figure 14. Moisture content through the insulation. The 6 bars for each layer represent data with and without dye, on days 1,2 and 3 . 


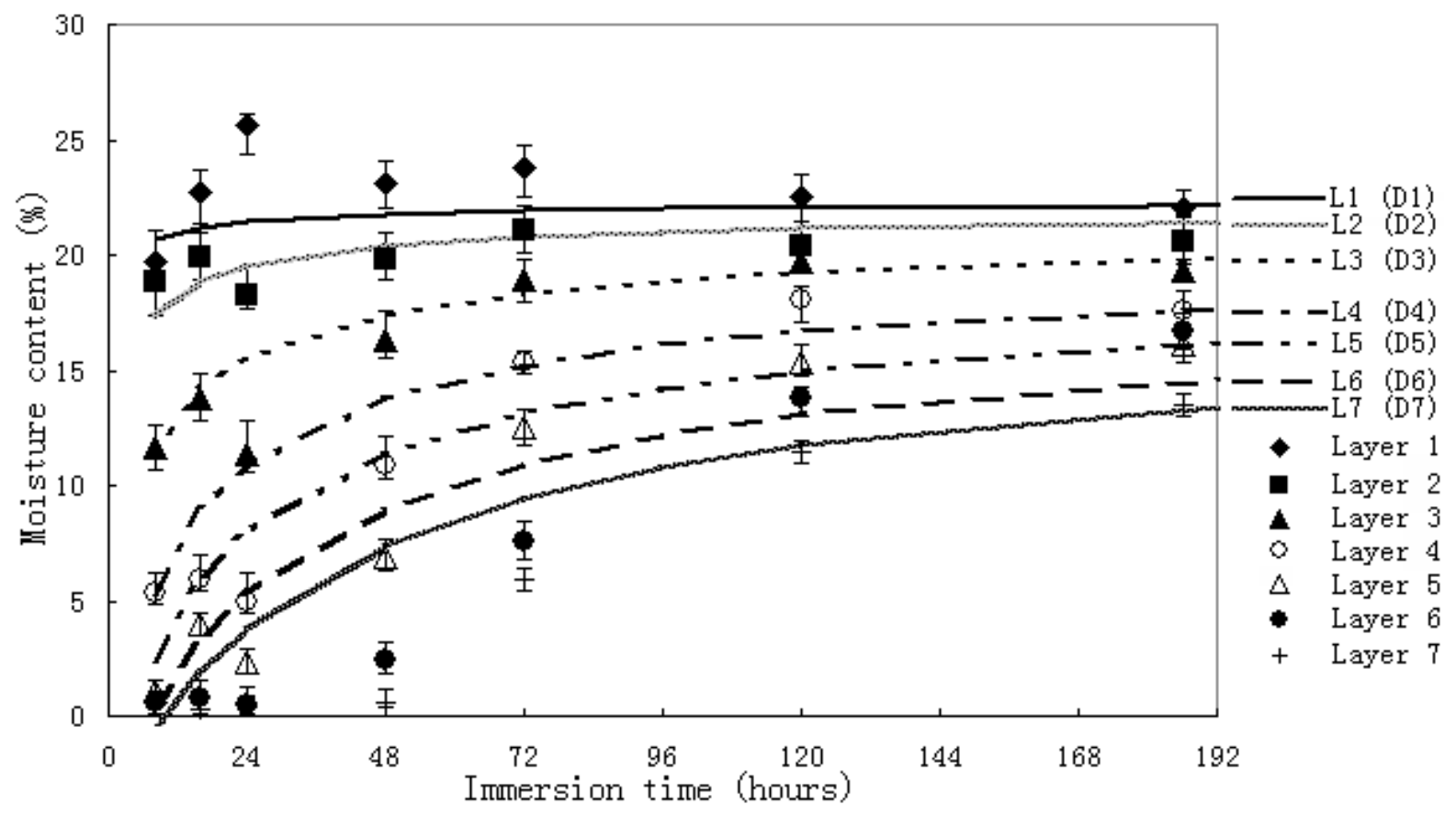

Figure 15. Moisture content as a function of time for each of 7 layers of insulation at room temperature. The average, and maximum and minimum recorded are depicted for each case. The data is fitted to equation 1 , with values of diffusion coefficient allowed to vary: $D$ (layer 1 ) $=8, D($ layer 2$)=4, D($ layer 3$)=1.5, D($ layer 4$)=0.8, D($ layer 5$)=0.7, D($ layer 6$)=0.6$, $D($ layer 7$)=0.6 . C_{s}=23$ in all cases. 


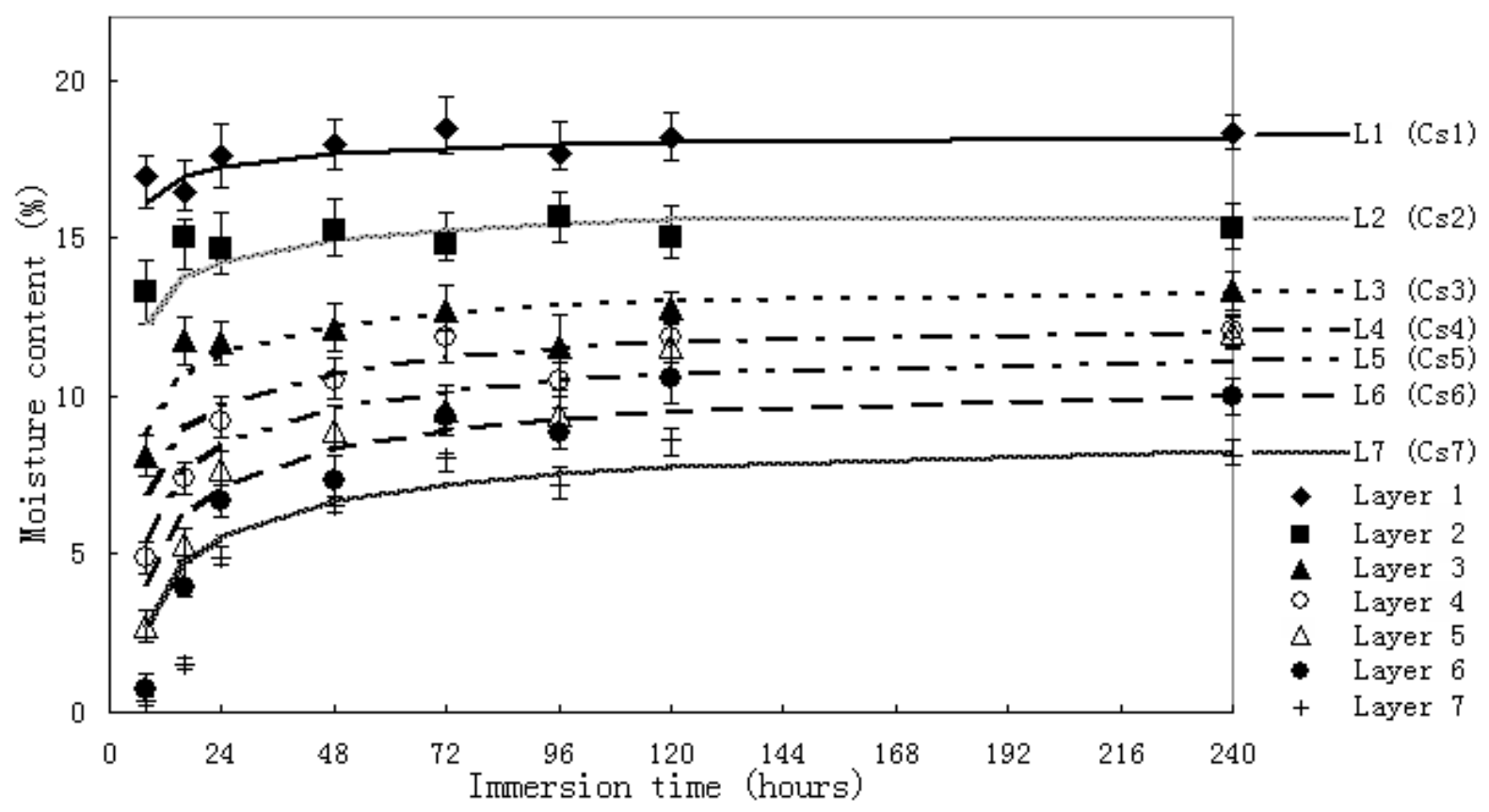

Figure 16. Moisture content as a function of time for each of 7 layers of insulation at $75^{\circ} \mathrm{C}$. The average, and maximum and minimum recorded are depicted for each case. The data is fitted to equation 1 , with value $C_{s}$ allowed to vary: $C_{s}($ layer 1$)=19, C_{s}($ layer 2$)=17, C_{s}($ layer 3$)=15$, $C_{s}($ layer 4$)=14, C_{s}($ layer 5$)=13, C_{s}($ layer 6$)=12, C_{s}($ layer 7$)=10.5$. In this case $D$ was fixed at 3.5 . 


\begin{tabular}{|c|c|c|c|c|}
\hline \multirow{2}{*}{$\begin{array}{c}\text { Temperature } \\
\left({ }^{\mathbf{0}} \mathbf{C}\right)\end{array}$} & \multicolumn{2}{|c|}{ Innermost layer } & \multicolumn{2}{c|}{ Outermost layer } \\
\cline { 2 - 5 } & Moisture content & Standard deviation & Moisture content & Standard deviation \\
\hline 20 & $22.7 \%$ & $1.20 \%$ & $23.8 \%$ & $1.23 \%$ \\
\hline 65 & $20.5 \%$ & $1.13 \%$ & $22.8 \%$ & $1.19 \%$ \\
\hline 75 & $20.0 \%$ & $1.16 \%$ & $22.1 \%$ & $1.17 \%$ \\
\hline
\end{tabular}

Table 1. Moisture uptake when paper is removed from the cable and soaked directly in water for 24 hours. The water was heated directly in these tests rather than the core.

\begin{tabular}{|c|c|c|c|c|}
\hline \multirow{2}{*}{$\begin{array}{c}\text { Temperature } \\
\left({ }^{\mathbf{0}} \mathbf{C}\right)\end{array}$} & \multicolumn{2}{|c|}{ Innermost layer } & \multicolumn{2}{c|}{ Outermost layer } \\
\cline { 2 - 5 } & Moisture content & Standard deviation & Moisture content & Standard deviation \\
\hline 20 & $20.6 \%$ & $1.16 \%$ & $23.0 \%$ & $1.18 \%$ \\
\hline 65 & $16.6 \%$ & $1.21 \%$ & $18.6 \%$ & $1.24 \%$ \\
\hline 75 & $15.0 \%$ & $1.14 \%$ & $17.7 \%$ & $1.20 \%$ \\
\hline
\end{tabular}

Table 2. Moisture uptake when paper is left on the cable and soaked directly in water after 24 hours. In one of the cases the outer six layers were removed to expose the inner layer. The water was heated directly in these tests. 